\title{
Resistive-wall impedance of two-layer tube
}

\author{
N. Wang* and Q. Qin ${ }^{\dagger}$ \\ Institute of High Energy Physics, 19(B) Yuquan Road, Beijing 100049, People's Republic of China
}

(Received 27 September 2007; published 28 November 2007)

\begin{abstract}
In contrast to electron machines, beam particles travel in most proton or heavy ion circular accelerators with nonrelativistic speed. The resistive-wall impedance of the cylindrical two-layer tube with finite thickness is studied in this paper for any material in the nonrelativistic case. The nonrelativistic correction for a special case of metallic-type layers is given afterward. Finally, we derive the general expression of the resistive-wall impedance for the multilayer tubes.
\end{abstract}

DOI: 10.1103/PhysRevSTAB.10.111003

PACS numbers: 29.27.Bd, 41.75.-i, 29.20.Dh

\section{INTRODUCTION}

Most modern proton or heavy ion accelerators are run or designed in nonrelativistic or moderate relativistic regions with high beam power. The impedance of vacuum chamber plays an important role for the stability of the circulating beams. As we all know, the wakefield generated by a beam of velocity $v$ smaller than the speed of light $c$ is different from that of the relativistic beam, and hence the impedance. Therefore the study of impedance and wakefield for the nonrelativistic beam is an important issue.

The resistive-wall impedance of a vacuum chamber for $v<c$ has been derived for a single layer tube with infinite [1,2] and finite wall thickness [3,4]. In Refs. [5-7], Ivanyan studied the longitudinal and transverse impedance for the two-layer tubes, and emphasized particularly on the relativistic beam impedance. Zotter and Kheifets studied the impedances and wakes of uniform charged beams in multilayer pipes [8].

In this paper, we will apply the field matching method to derive the general expression of the longitudinal and transverse impedances of a cylindrical two-layer tube. Then a special case of metallic-type layers is considered. The corresponding nonrelativistic corrections will also be derived. Finally, we will give a general expression for the multilayer tube with a finite thickness and arbitrary material.

\section{LONGITUDINAL IMPEDANCE OF TWO-LAYER TUBE}

The definition of impedance and wake function for the ultrarelativistic beam has been introduced in $[8,9]$. For the beam with an arbitrary velocity, we will use the generalized concept of wake and impedance. In the following, we will first derive the longitudinal impedance per unit length for the two-layer tube, and then the general result for the arbitrary multilayer will be given.

\footnotetext{
*wangn@ihep.ac.cn
}

†qinq@ihep.ac.cn
Let us consider a beam of line charge density $\lambda_{b} e^{i k s-i \omega t}$, where $\omega$ is angular frequency of beam and $k=\omega / v$, moving with a velocity $v$ along the axis of the beam pipe with an offset $a_{1}$. By using the definition of $z=(s-v t)$, every quantity $V$ is dependent on $s$ and $t$ as $V=\tilde{V} e^{i k z}$. The beam can be represented by a ring beam of radius $a_{1}$, whose charge density can be obtained from Eq. (1.7) in Ref. [9]:

$$
\rho_{0}(\vec{r}, z ; t)=\frac{\lambda_{b}}{2 \pi a_{1}} \delta\left(r-a_{1}\right) e^{i k z},
$$

and the corresponding axial current density

$$
\vec{j}_{0}(\vec{r}, z ; t)=\frac{\lambda_{b} v}{2 \pi a_{1}} \delta\left(r-a_{1}\right) e^{i k z} \hat{s} .
$$

Here we have adopted a cylindrical coordinate system $(r, \varphi, s)$, where $r, \varphi$, and $s$ are radial, azimuthal, and longitudinal coordinates, respectively.

The beam pipe can be divided into four regions as sketched in Fig. 1:

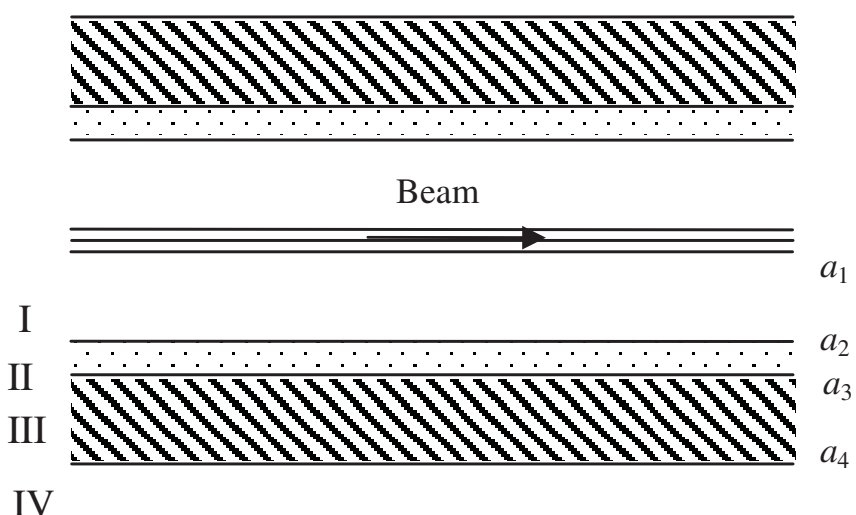

FIG. 1. Side view of the beam pipe. 


$$
\begin{cases}\text { (I) vacuum } & a_{1}<r<a_{2}, \\ \text { (II) 1st layer } & a_{2}<r<a_{3}, \\ \text { (III) 2nd layer } & a_{3}<r<a_{4}, \\ \text { (IV) vacuum } & r>a_{4},\end{cases}
$$

where $a_{i}(i=1,2,3$, and 4) are the radii of different layers.

Similar to that in Ref. [1], we use the wave equation of the scalar potential $\phi$ and magnetic vector potential $\vec{A}$ as the starting point of our calculation:

$$
\begin{gathered}
-\Delta \vec{A}+\frac{\mu_{r} \varepsilon_{r}}{c^{2}} \frac{\partial^{2} \vec{A}}{\partial t^{2}}+\mu_{0} \mu_{r} \sigma \frac{\partial \vec{A}}{\partial t}=\mu_{0} \mu_{r}(\vec{j}-\sigma \vec{E}), \\
-\Delta \phi+\frac{\mu_{r} \varepsilon_{r}}{c^{2}} \frac{\partial^{2} \phi}{\partial t^{2}}+\mu_{0} \mu_{r} \sigma \frac{\partial \phi}{\partial t}=\frac{\rho}{\varepsilon_{0} \varepsilon_{r}}
\end{gathered}
$$

where $\varepsilon_{r}$ is the relative dielectric constant, $\mu_{r}$ the relative magnetic permeability, $\sigma$ the conductivity for arbitrary medium, $j$ the current density, $\rho$ the charge density, and $E$ the electric field. $\varepsilon_{0}$ and $\mu_{0}$ are the dielectric constant and magnetic permeability of vacuum, respectively.

The relation of the electric and magnetic potentials is given by the Lorentz condition

$$
\left(\vec{\nabla} \cdot \vec{A}+\frac{\mu_{r} \varepsilon_{r}}{c^{2}} \frac{\partial \phi}{\partial t}+\mu_{0} \mu_{r} \sigma \phi\right)=0 .
$$

Let us consider that $j=0$ and $\sigma=0$ in the vacuum or insulated material, and Ohm's law $\vec{j}=\sigma \vec{E}$ exists in a medium with $\sigma \neq 0$. Additionally, $\rho$ is zero in the region outside the beam. Then the right-hand sides of Eqs. (3) and (4) are all zeros except at $r=a_{1}$. Because of the symmetry of the structure, the equation above can be simplified to

$$
\begin{aligned}
\frac{1}{r} \frac{\partial}{\partial r}\left(r \frac{\partial \tilde{A}_{s}}{\partial r}\right)-\left(k^{2}-\mu_{r} \varepsilon_{r} k_{0}^{2}-i \omega \mu_{0} \mu_{r} \sigma\right) \tilde{A}_{s} & =0, \\
\frac{1}{r} \frac{\partial}{\partial r}\left(r \frac{\partial \tilde{\phi}}{\partial r}\right)-\left(k^{2}-\mu_{r} \varepsilon_{r} k_{0}^{2}-i \omega \mu_{0} \mu_{r} \sigma\right) \tilde{\phi} & =0, \\
i k \tilde{A}_{s}-i \omega \frac{\mu_{r} \varepsilon_{r}}{c^{2}} \tilde{\phi}+\mu_{0} \mu_{r} \sigma \tilde{\phi} & =0 .
\end{aligned}
$$

By introducing

$$
\lambda^{2}=k^{2}-\mu_{r} \varepsilon_{r} k_{0}^{2}-i \omega \mu_{0} \mu_{r} \sigma,
$$

and the complex dielectric constant

$$
\varepsilon^{\prime}=\varepsilon_{r} \varepsilon_{0}+i \sigma / \omega,
$$

the general solution of the wave equations is given by

$$
\tilde{A}_{s}=p I_{0}(\lambda r)+q K_{0}(\lambda r), \quad \tilde{\phi}=\frac{k}{\omega \mu \varepsilon^{\prime}} \tilde{A}_{s} .
$$

Here $p$ and $q$ are unknown coefficients to be solved. This gives the nonvanishing magnetic and electric field

$$
\begin{aligned}
B_{\varphi} & =\left[-p \lambda I_{1}(\lambda r)+q \lambda K_{1}(\lambda r)\right] e^{i k z}, \\
E_{s} & =i \omega \frac{\lambda^{2}}{\lambda^{2}-k^{2}}\left[p I_{0}(\lambda r)+q K_{0}(\lambda r)\right] e^{i k z}, \\
E_{r} & =\omega \frac{\lambda k}{\lambda^{2}-k^{2}}\left[p I_{1}(\lambda r)-q K_{1}(\lambda r)\right] e^{i k z},
\end{aligned}
$$

where $I_{0}$ and $I_{1}$ are the modified Bessel functions of the first kind, and $K_{0}$ and $K_{1}$ are the modified Bessel functions of the second kind.

According to the wakefield expressions above, the tangential field can be written in the form of matrix

$$
\left(\begin{array}{c}
\tilde{H}_{\varphi} \\
\tilde{E}_{s}
\end{array}\right)_{i}=F\left(\lambda_{i} r\right)\left(\begin{array}{c}
p \\
q
\end{array}\right)_{i}
$$

where $i$ is the index of each layer of the chamber,

$$
F\left(\lambda_{i} r\right)=\left(\begin{array}{cc}
-b_{i} I_{1}\left(\lambda_{i} r\right) & b_{i} K_{1}\left(\lambda_{i} r\right) \\
c_{i} I_{0}\left(\lambda_{i} r\right) & c_{i} K_{0}\left(\lambda_{i} r\right)
\end{array}\right)
$$

and

$$
b_{i}=\lambda_{i} / \mu_{i}, \quad c_{i}=i \omega \frac{\lambda_{i}^{2}}{\lambda_{i}^{2}-k^{2}} .
$$

In order to obtain the unknown coefficients, we use the matching conditions of tangential field at the transition boundaries

$$
\left(\begin{array}{c}
\tilde{H}_{\varphi} \\
\tilde{E}_{s}
\end{array}\right)_{i}=\left(\begin{array}{c}
\tilde{H}_{\varphi} \\
\tilde{E}_{s}
\end{array}\right)_{i+1},
$$

i.e.,

$$
F\left(\lambda_{i} a_{i+1}\right)\left(\begin{array}{c}
p \\
q
\end{array}\right)_{i}=F\left(\lambda_{i+1} a_{i+1}\right)\left(\begin{array}{c}
p \\
q
\end{array}\right)_{i+1} .
$$

Then we can derive that

$$
\left(\begin{array}{l}
p \\
q
\end{array}\right)_{1}=\prod_{i=1}^{3} F^{-1}\left(\lambda_{i} a_{i+1}\right) F\left(\lambda_{i+1} a_{i+1}\right)\left(\begin{array}{l}
p \\
q
\end{array}\right)_{4},
$$

where

$$
F^{-1}\left(\lambda_{i} r\right)=-\frac{\lambda_{i} r}{b_{i} c_{i}}\left(\begin{array}{cc}
c_{i} K_{0}\left(\lambda_{i} r\right) & -b_{i} K_{1}\left(\lambda_{i} r\right) \\
-c_{i} I_{0}\left(\lambda_{i} r\right) & -b_{i} I_{1}\left(\lambda_{i} r\right)
\end{array}\right) .
$$

As the Bessel functions $I_{0}$ and $I_{1}$ diverge with increasing $r$, we have $p_{4}=0$. Additionally, the parameter $q_{1}$ is determined by the source boundary. In the beam region, we have $\lambda=k_{r}=\omega /(\gamma \beta c)$, and the wave equation for the vector potential $\tilde{A}_{s}$ satisfies

$$
\frac{1}{r} \frac{\partial}{\partial r}\left(r \frac{\partial \tilde{A}_{s}}{\partial r}\right)-k_{r}^{2} \tilde{A}_{s}=-\mu_{0} \tilde{j}_{0} .
$$

In order to solve the equation, we first derive the solution for beam source in the free space. Concerning that $\tilde{A}_{s}$ is continuous at $r=a_{1}$, the solution can be written as 


$$
\tilde{A}_{s}=\tilde{A}_{s 0} \begin{cases}I_{0}\left(k_{r} r\right) K_{0}\left(k_{r} a_{1}\right), & r \leq a_{1} \\ I_{0}\left(k_{r} a_{1}\right) K_{0}\left(k_{r} r\right), & r \geq a_{1},\end{cases}
$$

where $\tilde{A}_{s 0}$ is a coefficient to be determined. Notice that $I_{0}\left(k_{r} r\right)$ diverges with increasing $r$ and $K_{0}\left(k_{r} r\right)$ diverges as $r$ goes to zero. By integrating Eq. (18) over $r$ from $r=a_{1}-$ $\delta$ to $r=a_{1}+\delta$, where the parameter $\delta$ is infinitesimal, the left-hand side (lhs) of the integration turns to be $2 \pi \tilde{A}_{s 0} k_{r} a_{1}\left[-I_{0}\left(k_{r} a_{1}\right) K_{1}\left(k_{r} a_{1}\right)-I_{1}\left(k_{r} a_{1}\right) K_{0}\left(k_{r} a_{1}\right)\right]$, and the right-hand side (rhs) to be $-\mu_{0} \lambda_{b} v$. With the property of Bessel functions [10], the parenthesis on the lhs is $1 / k_{r} a_{1}$, and thus we can obtain

$$
\tilde{A}_{s 0}=\frac{\mu_{0} \lambda_{b} v}{2 \pi} .
$$

So in the region of $r \geq a_{1}$, we have

$$
q_{1}=\frac{\mu_{0} \lambda_{b} v}{2 \pi} I_{0}\left(k_{r} a_{1}\right), \quad \tilde{A}_{s}=\frac{\mu_{0} \lambda_{b} v}{2 \pi} I_{0}\left(k_{r} a_{1}\right) K_{0}\left(k_{r} r\right) .
$$

It should be mentioned that the expression of $\tilde{A}_{s}$ above is only applicable for the beam source in free space. When we impose the beam pipe boundary, a term proportional to $I_{0}\left(k_{r} r\right)$ should be added, as shown in Eq. (9).

So the only unknown coefficients are $p_{1}$ and $p_{4}$. Then we can obtain the ratio of $p_{1}$ and $q_{1}$ (see Appendix A), which is

$$
\frac{p_{1}}{q_{1}}=\frac{\kappa M K_{1}\left(k_{r} a_{2}\right)-K_{0}\left(k_{r} a_{2}\right)}{\kappa M I_{1}\left(k_{r} a_{2}\right)+I_{0}\left(k_{r} a_{2}\right)}
$$

with

$$
\kappa=-\frac{b_{1} c_{2}}{c_{1} b_{2}}
$$

$$
M=\frac{\left(S_{2,3} S_{3,1}+S_{2,4} S_{3,3}\right) K_{1}\left(k_{r} a_{4}\right)-\left(S_{2,3} S_{3,2}+S_{2,4} S_{3,4}\right) K_{0}\left(k_{r} a_{4}\right)}{\left(S_{2,1} S_{3,1}+S_{2,2} S_{3,3}\right) K_{1}\left(k_{r} a_{4}\right)-\left(S_{2,1} S_{3,2}+S_{2,2} S_{3,4}\right) K_{0}\left(k_{r} a_{4}\right)},
$$

and

$$
\begin{aligned}
& S_{i, 1}=c_{i} b_{i+1}\left[I_{1}\left(\lambda_{i} a_{i}\right) K_{0}\left(\lambda_{i} a_{i+1}\right)+K_{1}\left(\lambda_{i} a_{i}\right) I_{0}\left(\lambda_{i} a_{i+1}\right)\right], \\
& S_{i, 2}=b_{i} c_{i+1}\left[I_{1}\left(\lambda_{i} a_{i}\right) K_{1}\left(\lambda_{i} a_{i+1}\right)-K_{1}\left(\lambda_{i} a_{i}\right) I_{1}\left(\lambda_{i} a_{i+1}\right)\right], \\
& S_{i, 3}=c_{i} b_{i+1}\left[I_{0}\left(\lambda_{i} a_{i}\right) K_{0}\left(\lambda_{i} a_{i+1}\right)-K_{0}\left(\lambda_{i} a_{i}\right) I_{0}\left(\lambda_{i} a_{i+1}\right)\right], \\
& S_{i, 4}=b_{i} c_{i+1}\left[I_{0}\left(\lambda_{i} a_{i}\right) K_{1}\left(\lambda_{i} a_{i+1}\right)+K_{0}\left(\lambda_{i} a_{i}\right) I_{1}\left(\lambda_{i} a_{i+1}\right)\right] .
\end{aligned}
$$

Here, $i=2$ and 3 . The vector potential is

$$
\begin{aligned}
\tilde{A}_{s}= & \frac{\mu_{0} \lambda_{b} \nu}{2 \pi} I_{0}\left(k_{r} a_{1}\right)\left[K_{0}\left(k_{r} r\right)+I_{0}\left(k_{r} r\right)\right. \\
& \left.\times \frac{\kappa M K_{1}\left(k_{r} a_{2}\right)-K_{0}\left(k_{r} a_{2}\right)}{\kappa M I_{1}\left(k_{r} a_{2}\right)+I_{0}\left(k_{r} a_{2}\right)}\right] .
\end{aligned}
$$

Following Eq. (2.64) in [9] and $J_{0}=\lambda_{b} v$, the longitudinal impedance per unit length becomes

$$
\begin{aligned}
Z_{\|}(\omega)= & \frac{i Z_{0} c k_{r}^{2}}{2 \pi \omega} I_{0}\left(k_{r} a_{1}\right)\left[K_{0}\left(k_{r} r\right)+I_{0}\left(k_{r} r\right)\right. \\
& \left.\times \frac{\kappa M K_{1}\left(k_{r} a_{2}\right)-K_{0}\left(k_{r} a_{2}\right)}{\kappa M I_{1}\left(k_{r} a_{2}\right)+I_{0}\left(k_{r} a_{2}\right)}\right],
\end{aligned}
$$

where $Z_{0}=\mu_{0} c \approx 377 \Omega$ is the impedance of free space.

Until now, we have obtained the exact expression of the longitudinal impedance of a two-layer tube. It has a similar form as that obtained by Zimmermann and Oide for single layer beam pipe with infinite thickness. As we will see later, the longitudinal impedance of any multilayer tubes can be written in the form of Eq. (27), except for the different expression of $M$.

It is easy to show that, for the point beam source moving on-axis of the beam pipe, all the corresponding results can be obtained by removing the factor of $I_{0}\left(k_{r} a_{1}\right)$.

\section{TRANSVERSE IMPEDANCE OF TWO-LAYER TUBE}

In the previous section we obtained the longitudinal impedance of monopole mode with the method of field matching. The transverse impedance can be obtained using the Panofsky-Wenzel theorem [11], i.e., one has to obtain the longitudinal impedance of the dipole mode first.

Considering a beam source with dipole line density of $\lambda_{b} d e^{i k z}$, the equivalent charge and current density can be expressed as

$$
\begin{gathered}
\rho_{1}(\vec{r}, z ; t)=\frac{\lambda_{b} d}{\pi a_{1}^{2}} \delta\left(r-a_{1}\right) \cos \varphi e^{i k z}, \\
\vec{j}_{1}(\vec{r}, z ; t)=\beta c \rho_{1} \hat{s} .
\end{gathered}
$$

Here we suppose $d=2 a_{1}$ is the distance of the charges in the dipole.

First, we derive the dipole electromagnetic field generated by the beam source described above in free space. For the dipole moment, the wave equation for the vector potential is [see Eq. (67) in Ref. [1]]

$$
\frac{1}{r} \frac{\partial}{\partial r}\left(r \frac{\partial \tilde{A}_{s}}{\partial r}\right)-\left(\frac{1}{r^{2}}+k_{r}^{2}\right) \tilde{A}_{s}=-\mu_{0} \tilde{j}_{s}
$$


Done with the same analyses as the longitudinal case, the solution of $\tilde{A}_{s}$ can be written as

$$
\tilde{A}_{s}=\tilde{A}_{s 1} \cos \varphi \begin{cases}I_{1}\left(k_{r} r\right) K_{1}\left(k_{r} a_{1}\right), & r \leq a_{1} \\ I_{1}\left(k_{r} a_{1}\right) K_{1}\left(k_{r} r\right), & r \geq a_{1},\end{cases}
$$

where $\tilde{A}_{s 1}$ is an unknown coefficient. Integrating Eq. (30) over $r$ from $r=a_{1}-\delta$ to $r=a_{1}+\delta$, we can find the lhs of the equation turns to

lhs $=\tilde{A}_{s 1} k_{r} a_{1}\left(I_{1}\left(k_{r} a_{1}\right) K_{1}^{\prime}\left(k_{r} a_{1}\right)-I_{1}^{\prime}\left(k_{r} a_{1}\right) K_{1}\left(k_{r} a_{1}\right)\right) \cos \varphi$,

and the rhs is

$$
\mathrm{rhs}=-\frac{\mu_{0}}{\pi a_{1}} \lambda_{b} d v \cos \varphi
$$

So we can obtain the coefficient $\tilde{A}_{s 1}$, which is

$$
\tilde{A}_{s 1}=\frac{\mu_{0}}{\pi a_{1}} \lambda_{b} d v
$$

and the vector potential in the region $r \geq a_{1}$ is

$$
\tilde{A}_{s}=\frac{\mu_{0}}{\pi a_{1}} \lambda_{b} d v \cos \varphi I_{1}\left(k_{r} a_{1}\right) K_{1}\left(k_{r} r\right) .
$$

Here the properties of Bessel functions have also been used. Similar to the longitudinal case, the solutions correspond to the beam source in free space, and the solution with beam pipe boundary should be added by a term of $I_{1}\left(k_{r} r\right)$.

We obtain the expressions for the field components $E_{s}$, $E_{\varphi}, B_{\varphi}$, and $B_{s}$ by repeating the calculation process in Ref. [1], except that there is a factor of $1 / 2$ missing in the expressions $A_{r}$ and $A_{\varphi}$ of Eq. (82) in Ref. [1] and the argument of Bessel functions is replaced by $\lambda_{i} r$. The electric and magnetic field strengths can be written as

$$
\begin{aligned}
B_{\varphi}= & \left(\frac{i k}{2}\left[p_{+}\left(I_{2}-I_{0}\right)+q_{+}\left(K_{2}-K_{0}\right)\right]\right. \\
& \left.-\frac{\lambda}{2}\left[p_{s}\left(I_{0}+I_{2}\right)-q_{s}\left(K_{0}+K_{2}\right)\right]\right) \cos \varphi e^{i k z}, \\
B_{s}= & \lambda\left(p_{+} I_{1}-q_{+} K_{1}\right) \sin \varphi e^{i k z}, \\
E_{\varphi}= & {\left[\frac{i \omega}{2}\left[p_{+}\left(I_{2}+I_{0}\right)+q_{+}\left(K_{2}+K_{0}\right)\right]\right.} \\
& \left.+\frac{1}{r} \frac{k \omega}{k^{2}-\lambda^{2}}\left(p_{s} I_{1}+q_{s} K_{1}\right)\right] \sin \varphi e^{i k z}, \\
E_{s}= & -\frac{i \omega \lambda^{2}}{k^{2}-\lambda^{2}}\left(p_{s} I_{1}+q_{s} K_{1}\right) \cos \varphi e^{i k z} .
\end{aligned}
$$

Here we use a gauge transformation combining with the Lorentz condition. The gauge function is taken to be $\chi=$ $\left[\left(p_{+}+p_{-}\right) I_{1}(\lambda r)+\left(q_{+}+q_{-}\right) K_{1}(\lambda r)\right] / \lambda e^{i k z} \cos \varphi, \quad$ and the following restrictions have been used:

$$
\begin{array}{cl}
p_{+}=-p_{-}, & q_{+}=-q_{-}, \\
p_{s}=\frac{k^{2}-\lambda^{2}}{k \omega} p_{0}, & q_{s}=\frac{k^{2}-\lambda^{2}}{k \omega} q_{0} .
\end{array}
$$

So the unknown coefficients in the wakefield expressions are determined by the following matching conditions:

$$
G\left(\lambda_{i} a_{i+1}\right)\left(\begin{array}{c}
p_{+} \\
p_{s} \\
q_{+} \\
q_{s}
\end{array}\right)_{i}=G\left(\lambda_{i+1} a_{i+1}\right)\left(\begin{array}{c}
p_{+} \\
p_{s} \\
q_{+} \\
q_{s}
\end{array}\right)_{i+1},
$$

where $G(\lambda r)$ is given by

$$
G(\lambda r)=\left(\begin{array}{cccc}
-\frac{i k}{\mu \lambda r} I_{1} & -\frac{\lambda}{\mu} I_{1}^{\prime} & \frac{i k}{\mu \lambda r} K_{1} & -\frac{\lambda}{\mu} K_{1}^{\prime} \\
\frac{\lambda}{\mu} I_{1} & 0 & -\frac{\lambda}{\mu} K_{1} & 0 \\
i \omega I_{1}^{\prime} & \frac{1}{r} \frac{k \omega}{k^{2}-\lambda^{2}} I_{1} & -i \omega K_{1}^{\prime} & \frac{1}{r} \frac{k \omega}{k^{2}-\lambda^{2}} K_{1} \\
0 & -\frac{i \omega \lambda^{2}}{k^{2}-\lambda^{2}} I_{1} & 0 & -\frac{i \omega \lambda^{2}}{k^{2}-\lambda^{2}} K_{1}
\end{array}\right),
$$

and $I^{\prime}$ and $K^{\prime}$ denote the derivation of the modified Bessel functions, which have the argument of $\lambda r$.

According to the source boundary presented at the beginning of this section, we have

$$
\begin{gathered}
q_{s 1}=\frac{\mu_{0}}{\pi a_{1}} \lambda_{b} d v I_{1}\left(k_{r} a_{1}\right), \quad q_{01}=\frac{c^{2} k}{\omega} q_{s 1}, \\
q_{+1}=0, \quad q_{-1}=0 .
\end{gathered}
$$

The convergence of Bessel function $I(\lambda r)$ gives $p_{04}=$ $p_{+4}=0$. Applying the conditions above to Eq. (38), we have

$\left(\begin{array}{c}p_{+} \\ p_{s} \\ 0 \\ q_{s}\end{array}\right)_{1}=\prod_{i=1}^{3} G^{-1}\left(\lambda_{i} a_{i+1}\right) G\left(\lambda_{i+1} a_{i+1}\right)\left(\begin{array}{c}0 \\ 0 \\ q_{+} \\ q_{s}\end{array}\right)_{4}$

where $G^{-1}$ is the inverse matrix of $G$, and

$$
G^{-1}(\lambda r)=\left(\begin{array}{cccc}
0 & -\mu r K_{1}^{\prime} & -\frac{i \lambda r}{\omega} K_{1} & -\frac{k}{\lambda \omega} K_{1} \\
-\mu r K_{1} & -\frac{i \mu k}{\lambda^{2}} K_{1} & 0 & -\frac{i\left(k^{2}-\lambda^{2}\right) r}{\lambda \omega} K_{1}^{\prime} \\
0 & -\mu r I_{1}^{\prime} & -\frac{i \lambda r}{\omega} I_{1} & -\frac{k}{\lambda \omega} I_{1} \\
\mu r I_{1} & \frac{i \mu k}{\lambda^{2}} I_{1} & 0 & \frac{i\left(k^{2}-\lambda^{2}\right) r}{\lambda \omega} I_{1}^{\prime}
\end{array}\right)
$$


In deriving the inverse matrix of $G$, we have used the property of Bessel functions [10]:

$$
I_{p}(x) K_{p+1}(x)+I_{p+1}(x) K_{p}(x)=\frac{1}{x} .
$$

For simplicity, we express the $4 \times 4$ matrices of $G$ and $G^{-1}$ in the $2 \times 2$ block form as

$$
\begin{aligned}
G(\lambda r) & =\left(\begin{array}{ll}
A(\lambda r) & B(\lambda r) \\
C(\lambda r) & D(\lambda r)
\end{array}\right), \\
G^{-1}(\lambda r) & =\left(\begin{array}{ll}
\xi B^{*}(\lambda r) & \zeta D^{*}(\lambda r) \\
\xi A^{*}(\lambda r) & \zeta C^{*}(\lambda r)
\end{array}\right),
\end{aligned}
$$

where

$$
\begin{aligned}
& A(\lambda r)=\left(\begin{array}{cc}
-\frac{i k I_{1}}{\mu \lambda r} & -\frac{\lambda I_{1}^{\prime}}{\mu} \\
\frac{\lambda I_{1}}{\mu} & 0
\end{array}\right), \\
& B(\lambda r)=\left(\begin{array}{cc}
\frac{i k K_{1}}{\mu \lambda r} & -\frac{\lambda K_{1}^{\prime}}{\mu} \\
-\frac{\lambda K_{1}}{\mu} & 0
\end{array}\right), \\
& C(\lambda r)=\left(\begin{array}{cc}
i \omega I_{1}^{\prime} & \frac{1}{r} \frac{k \omega I_{1}}{k^{2}-\lambda^{2}} \\
0 & -\frac{i \omega \lambda^{2} I_{1}}{k^{2}-\lambda^{2}}
\end{array}\right), \\
& D(\lambda r)=\left(\begin{array}{cc}
-i \omega K_{1}^{\prime} & \frac{1}{r} \frac{k \omega K_{1}}{k^{2}-\lambda^{2}} \\
0 & -\frac{i \omega \lambda^{2} K_{1}}{k^{2}-\lambda^{2}}
\end{array}\right),
\end{aligned}
$$

and $A^{*}=\operatorname{det}(A) A^{-1}, B^{*}=\operatorname{det}(B) B^{-1}, C^{*}=\operatorname{det}(C) C^{-1}$, $D^{*}=\operatorname{det}(D) D^{-1}, \xi=-\mu^{2} r / \lambda, \zeta=\left(k^{2}-\lambda^{2}\right) r /\left(\omega^{2} \lambda\right)$.

Then Eq. (41) turns to be

$$
\begin{aligned}
\left(\begin{array}{c}
p_{+} \\
p_{s} \\
0 \\
q_{s}
\end{array}\right)_{1}= & \left(\begin{array}{ll}
\xi_{1} B^{*}\left(k_{r} a_{2}\right) & \zeta_{1} D^{*}\left(k_{r} a_{2}\right) \\
\xi_{1} A^{*}\left(k_{r} a_{2}\right) & \zeta_{1} C^{*}\left(k_{r} a_{2}\right)
\end{array}\right)\left(\begin{array}{ll}
L_{11}^{(2)} & L_{12}^{(2)} \\
L_{21}{ }^{(2)} & L_{22}^{(2)}
\end{array}\right) \\
& \times\left(\begin{array}{ll}
L_{11}^{(3)} & L_{12}^{(3)} \\
L_{21}^{(3)} & L_{22}^{(3)}
\end{array}\right)\left(\begin{array}{ll}
A\left(k_{r} a_{4}\right) & B\left(k_{r} a_{4}\right) \\
C\left(k_{r} a_{4}\right) & D\left(k_{r} a_{4}\right)
\end{array}\right) \\
& \times\left(\begin{array}{c}
0 \\
0 \\
q_{+} \\
q_{s}
\end{array}\right)_{4}
\end{aligned}
$$

where matrices $\left(L_{i j}\right)$ 's are defined by Eq. (B4). The solution will be (see Appendix B)

$$
\begin{aligned}
\left(\begin{array}{c}
p_{+} \\
p_{s}
\end{array}\right)_{1}= & {\left[\xi_{1} B^{*}\left(k_{r} a_{2}\right) N_{1}+\zeta_{1} D^{*}\left(k_{r} a_{2}\right) N_{2}\right] } \\
& \times\left[\xi_{1} A^{*}\left(k_{r} a_{2}\right) N_{1}+\zeta_{1} C^{*}\left(k_{r} a_{2}\right) N_{2}\right]^{-1}\left(\begin{array}{c}
0 \\
q_{s}
\end{array}\right)_{1},
\end{aligned}
$$

and

$$
\begin{aligned}
& N_{1}=\left(\begin{array}{ll}
L_{11}{ }^{(2)} & L_{12}{ }^{(2)}
\end{array}\right)\left(\begin{array}{ll}
L_{11}{ }^{(3)} & L_{12}{ }^{(3)} \\
L_{21}{ }^{(3)} & L_{22}{ }^{(3)}
\end{array}\right)\left(\begin{array}{c}
B\left(k_{r} a_{4}\right) \\
D\left(k_{r} a_{4}\right)
\end{array}\right), \\
& N_{2}=\left(\begin{array}{ll}
L_{21}{ }^{(2)} & L_{22}{ }^{(2)}
\end{array}\right)\left(\begin{array}{ll}
L_{11}{ }^{(3)} & L_{12}{ }^{(3)} \\
L_{21}{ }^{(3)} & L_{22}{ }^{(3)}
\end{array}\right)\left(\begin{array}{c}
B\left(k_{r} a_{4}\right) \\
D\left(k_{r} a_{4}\right)
\end{array}\right) .
\end{aligned}
$$

By expanding the matrices in Eq. (47), we can easily get the ratio of $p_{s 1} / q_{s 1}$, which will be a very complex expression. According to Eq. (36), one can obtain the longitudinal impedance of dipole mode as

$$
\begin{aligned}
Z_{\|}^{(1)}(\omega) & =-\frac{E_{s}}{d \lambda_{b} v r \cos \varphi e^{i k z}} \\
& =\frac{q_{s 1}}{d \lambda_{b} v r} \frac{i c^{2} k_{r}^{2}}{\omega}\left(\frac{p_{s 1}}{q_{s 1}} I_{1}\left(k_{r} r\right)+K_{1}\left(k_{r} r\right)\right) .
\end{aligned}
$$

The transverse impedance can be derived from the Panofsky-Wenzel theorem.

Similar to the longitudinal treatments, we can get the results for the point beam source on-axis by expanding the factor of $I_{1}\left(k_{r} a_{1}\right)$ as $\frac{k_{r} a_{1}}{2}\left(1+\frac{k_{r}^{2} a_{1}^{2}}{8}+\cdots\right)$.

\section{SPECIAL CASE OF METALLIC LAYERS}

For metallic layers of a resistive-wall impedance, it is reasonable to take the conception that the beam aperture is much larger than the skin depth $\delta_{2,3}$, which means $\left|\lambda_{2} r\right| \gg$ 1 and $\left|\lambda_{3} r\right| \gg 1$. So we can assume that

$$
\begin{aligned}
& \lambda_{2} \approx \lambda_{20} \approx \frac{1-i \operatorname{sgn}(\omega)}{\delta_{2}}, \\
& \lambda_{3} \approx \lambda_{30} \approx \frac{1-i \operatorname{sgn}(\omega)}{\delta_{3}},
\end{aligned}
$$

and the Bessel functions can be approximated by [10]

$$
\begin{array}{cc}
K_{p}(x) \sim \sqrt{\frac{\pi}{2 x} e^{-x}} & (p \geq 0, x \rightarrow \infty), \\
I_{p}(x) \sim \frac{1}{\sqrt{2 \pi x}} e^{x} & (p \geq 0, x \rightarrow \infty) .
\end{array}
$$

First, we take the above approximations to the longitudinal impedance expressed as Eq. (27), and the parameters $\kappa$ and $M$ can be simplified as

$$
\kappa=-\frac{\lambda_{2}}{k_{r} \mu_{0} \varepsilon_{2}^{\prime} c^{2}},
$$

and 


$$
M=-\frac{\varepsilon_{0} \lambda_{3}\left[\alpha \tanh \left(x_{1}\right)+\tanh \left(x_{2}\right)\right] K_{1}\left(k_{r} a_{4}\right)+\varepsilon_{3}^{\prime} k_{r}\left[\alpha \tanh \left(x_{1}\right) \tanh \left(x_{2}\right)+1\right] K_{0}\left(k_{r} a_{4}\right)}{\varepsilon_{0} \lambda_{3}\left[\alpha+\tanh \left(x_{1}\right) \tanh \left(x_{2}\right)\right] K_{1}\left(k_{r} a_{4}\right)+\varepsilon_{3}^{\prime} k_{r}\left[\alpha \tanh \left(x_{2}\right)+\tanh \left(x_{1}\right)\right] K_{0}\left(k_{r} a_{4}\right)}
$$

where $\quad \alpha=\left(\lambda_{2} \varepsilon_{3}^{\prime}\right) /\left(\varepsilon_{2}^{\prime} \lambda_{3}\right) \approx\left(\delta_{2} \mu_{2}\right) /\left(\delta_{3} \mu_{3}\right), \quad x_{1}=$ $\lambda_{2}\left(a_{3}-a_{2}\right)$, and $x_{2}=\lambda_{3}\left(a_{4}-a_{3}\right)$.

For $k_{r} r \ll 1$, we simplify $Z_{\|}(\omega)$ to the first order of $\delta_{\text {skin }} \omega / c$ and the second order of $k_{r} / k_{0}$, keeping the term $\delta_{\text {skin }} k_{r}^{2}$, the longitudinal impedance is then

$$
\begin{aligned}
Z_{\|}(\omega)= & \frac{Z_{0}}{2 \pi c}\left[-\frac{i c^{2} k_{r}^{2}}{\omega} \ln \left(\frac{r}{a_{2}}\right)-\frac{\delta_{2} \mu_{2} \omega[\operatorname{sgn}(\omega)-i]}{2 a_{2} \mu_{0}}\right. \\
& \left.\times\left(1-\frac{k_{r}^{2}\left(2 a_{2}^{2}-r^{2}-a_{1}^{2}\right)}{4}\right) M\right]
\end{aligned}
$$

The first term in the parentheses of the rhs of Eq. (54) is the space charge impedance, which is the same as that in Ref. [1]. The second one is supposed to be the resistivewall impedance of the two-layer tube, i.e.,

$$
\begin{aligned}
Z_{\|, r w}(\omega)= & \frac{Z_{0}}{2 \pi c}\left[-\frac{\delta_{2} \mu_{2} \omega[\operatorname{sgn}(\omega)-i]}{2 a_{2} \mu_{0}}\right. \\
& \left.\times\left(1-\frac{k_{r}^{2}\left(2 a_{2}^{2}-r^{2}-a_{1}^{2}\right)}{4}\right) M\right] .
\end{aligned}
$$

For the ultrarelativistic case with $v=c$ and $k_{r} \rightarrow 0$, the above expression turns to be

$$
\begin{aligned}
Z_{\|, r w}(\omega)= & \frac{Z_{0}}{2 \pi c}\left[\frac{\delta_{2} \mu_{2} \omega[\operatorname{sgn}(\omega)-i]}{2 a_{2} \mu_{0}}\right. \\
& \left.\times \frac{\alpha \tanh \left(x_{1}\right)+\tanh \left(x_{2}\right)}{\alpha+\tanh \left(x_{1}\right) \tanh \left(x_{2}\right)}\right],
\end{aligned}
$$

which is consistent with the result obtained in Ref. [5] if we assume that the first term of Eq. (19) in Ref. [5] is much smaller than the second one, which is always correct for metal layers. For the single layer tube with infinite thickness, i.e., $\lambda_{2}=\lambda_{3}, \varepsilon_{2}^{\prime}=\varepsilon_{3}^{\prime}$, and $a_{4} \rightarrow \infty$, Eq. (55) is reduced to Eq. (57) in Ref. [1] if we have $a_{1} \rightarrow 0$ for the on-axis beam source. It also demonstrates that, for the same zeroth moment of the beam, the impedance is independent of the detailed shape of the beam distribution in the ultrarelativistic limit [9], but this is no longer true for the nonrelativistic or moderate relativistic beam.

Correspondingly, in order to obtain the transverse impedance, we first derive the longitudinal impedance of the dipole mode, which can be obtained by substituting Eqs. (50) and (51) into Eq. (49). The result is much more complicated than that of the monopole mode. Through further expanding the expression to the first order of $\delta_{\text {skin }} \omega / c$ and the second order of $k_{r} / k_{0}$, and for the nonrelativistic case we always have $\delta_{\text {skin }}<k_{r}^{2}$, the longitudinal electric field becomes

$$
\begin{aligned}
E_{s}= & \frac{i c^{2} k_{r}^{2} q_{s}}{\omega}\left(\frac{\left(r^{2}-a_{2}^{2}\right)}{k_{r} a_{2}^{2} r}+\delta_{2} \frac{1+i \operatorname{sgn}(\omega)}{2} \frac{\omega^{2} \mu_{2} r}{2 a_{2}^{3} c^{2} k_{r}^{3} \mu_{0}}\right. \\
& \left.\times\left(4-k_{r}^{2} a_{2}^{2}+\frac{k_{r}^{2} r^{2}}{2}\right) \frac{1+\alpha \tanh \left(x_{1}\right) \tanh \left(x_{2}\right)}{\alpha \tanh \left(x_{2}\right)+\tanh \left(x_{1}\right)}\right) \\
& \times \cos \varphi e^{i k z} .
\end{aligned}
$$

The longitudinal impedance of the dipole mode is then

$$
\begin{aligned}
Z_{\|}^{(1)}(\omega)= & -i \frac{Z_{0}}{c}\left(\frac{c^{2} k_{r}^{2}\left(r^{2}-a_{2}^{2}\right)}{2 \pi a_{2}^{2} \omega r^{2}}+\delta_{2} \frac{1+i \operatorname{sgn}(\omega)}{2}\right. \\
& \times \frac{\omega \mu_{2}}{4 \pi a_{2}^{3} \mu_{0}}\left(4-k_{r}^{2} a_{2}^{2}+\frac{1}{2} k_{r}^{2} r^{2}+\frac{1}{2} k_{r}^{2} a_{1}^{2}\right) \\
& \left.\times \frac{1+\alpha \tanh \left(x_{1}\right) \tanh \left(x_{2}\right)}{\alpha \tanh \left(x_{2}\right)+\tanh \left(x_{1}\right)}\right)
\end{aligned}
$$

The first term in Eq. (58) is independent of skin depth and equals the case of the one-layer tube [see Eq. (121) in Ref. [1]] with $b=a_{2}$ and $a_{1} \rightarrow 0$, which is defined as the space charge impedance. The second term is the longitudinal resistive-wall impedance of the dipole mode.

Then the transverse impedance for the resistive wall can be obtained according to the Panofsky-Wenzel theorem:

$$
\begin{aligned}
Z_{\perp, r w}(\omega)= & \frac{Z_{\|, r w}^{(1)}(\omega)}{k} \\
\approx & \frac{1}{\sqrt{\omega^{2} / c^{2}+k_{r}^{2}}} \frac{1-i \operatorname{sgn}(\omega)}{4 \pi a_{2}^{3} \delta_{2} \sigma_{2}} \\
& \times\left(4-k_{r}^{2} a_{2}^{2}+\frac{1}{2} k_{r}^{2} r^{2}+\frac{1}{2} k_{r}^{2} a_{1}^{2}\right) \\
& \times \frac{1+\alpha \tanh \left(x_{1}\right) \tanh \left(x_{2}\right)}{\alpha \tanh \left(x_{2}\right)+\tanh \left(x_{1}\right)},
\end{aligned}
$$

where $\alpha, x_{1}$, and $x_{2}$ have the same definitions as those in Eq. (53). For the case of the single layer tube with infinite thickness, the fraction part containing $x_{1}$ and $x_{2}$ is reduced to 1 , and Eq. (59) becomes

$$
\begin{aligned}
Z_{\perp, r w}(\omega)= & \frac{Z_{\|, r w}^{(1)}(\omega)}{k} \\
\approx & \frac{1}{\sqrt{\omega^{2} / c^{2}+k_{r}^{2}}} \frac{1-i \operatorname{sgn}(\omega)}{4 \pi a_{2}^{3} \delta_{2} \sigma_{2}} \\
& \times\left(4-k_{r}^{2} a_{2}^{2}+\frac{1}{2} k_{r}^{2} r^{2}+\frac{1}{2} k_{r}^{2} a_{1}^{2}\right),
\end{aligned}
$$

which is consistent with Eq. (124) in Ref. [1] when $a_{1} \rightarrow 0$.

The transverse impedance for the relativistic case can be obtained using the similar method if we consider $k_{r}^{2}<$ $\delta_{\text {skin }}$. 


\section{IMPEDANCE OF MULTILAYER TUBE}

According to the impedance calculation of two-layer tube, we can generalize the expression to describe the longitudinal and transverse impedances for a multilayer tube. For the longitudinal impedance, the unknown parameter $p_{1}$ can be derived from the equation below as described in Eq. (16):

$$
\left(\begin{array}{l}
p \\
q
\end{array}\right)_{1}=\prod_{i=1}^{n-1} F^{-1}\left(\lambda_{i} a_{i+1}\right) F\left(\lambda_{i+1} a_{i+1}\right)\left(\begin{array}{c}
p \\
q
\end{array}\right)_{n},
$$

where $F$ and $F^{-1}$ are given by Eqs. (12) and (17), respectively, and $n>1$ is the number of regions in the structure including the vacuum area.

The longitudinal monopole impedance can then be expressed as a function of $M$ (see Appendix A):

$$
\begin{aligned}
Z_{\|}(\omega)= & \frac{i Z_{0} c k_{r}^{2}}{2 \pi \omega} I_{0}\left(k_{r} a_{1}\right)\left[K_{0}\left(k_{r} r\right)+I_{0}\left(k_{r} r\right)\right. \\
& \left.\times \frac{\kappa M K_{1}\left(k_{r} a_{2}\right)-K_{0}\left(k_{r} a_{2}\right)}{\kappa M I_{1}\left(k_{r} a_{2}\right)+I_{0}\left(k_{r} a_{2}\right)}\right],
\end{aligned}
$$

where $\kappa$ and $M$ are defined as

$$
\begin{aligned}
& \kappa=-\frac{b_{1} c_{2}}{c_{1} b_{2}}, \\
& M=\left(\begin{array}{ll}
S_{1,3} & S_{1,4}
\end{array}\right) \prod_{i=2}^{n-1}\left(\begin{array}{ll}
S_{i, 1} & S_{i, 2} \\
S_{i, 3} & S_{i, 4}
\end{array}\right)\left(\begin{array}{c}
K_{1}(\lambda a) \\
-K_{0}(\lambda a)
\end{array}\right)_{n} \\
&\left(\begin{array}{ll}
S_{1,1} & S_{1,2}
\end{array}\right) \prod_{i=2}^{n-1}\left(\begin{array}{cc}
S_{i, 1} & S_{i, 2} \\
S_{i, 3} & S_{i, 4}
\end{array}\right)\left(\begin{array}{c}
K_{1}(\lambda a) \\
-K_{0}(\lambda a)
\end{array}\right)_{n}
\end{aligned}
$$

with $S$ 's for $i>1$ given by Eq. (25), and for $i=1$,

$$
\left(\begin{array}{ll}
S_{1,1} & S_{1,2} \\
S_{1,3} & S_{1,4}
\end{array}\right)=\left(\begin{array}{ll}
1 & 0 \\
0 & 1
\end{array}\right)
$$

According to the expressions we get above, we can tell that the impedance is mainly determined by the parameters of the first layer except the parameter $M$, which is dependent on every layer.

Correspondingly, for the case of metallic multilayers, the longitudinal impedance can be simplified to

$$
\begin{aligned}
Z_{\|}(\omega)= & \frac{Z_{0}}{2 \pi c}\left[-\frac{i c^{2} k_{r}^{2}}{\omega} \ln \left(\frac{r}{a_{2}}\right)+\frac{\delta_{2} \mu_{2} \omega[\operatorname{sgn}(\omega)-i]}{2 a_{2} \mu_{0}}\right. \\
& \left.\times\left(1-\frac{k_{r}^{2}\left(2 a_{2}^{2}-r^{2}-a_{1}^{2}\right)}{4}\right) M\right],
\end{aligned}
$$

with

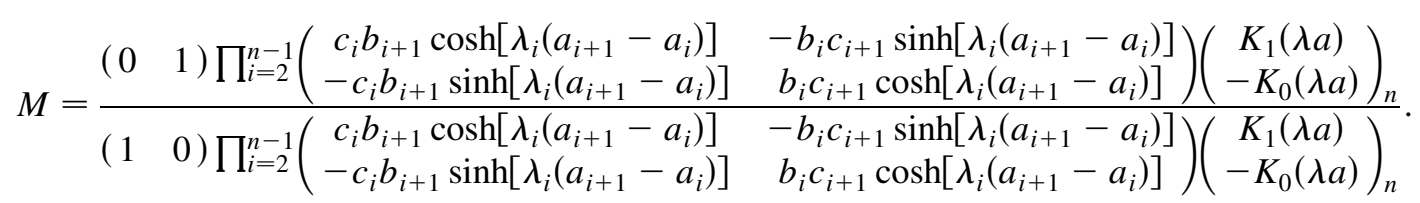

The first term of Eq. (65) is the space charge impedance, and the second one the resistive-wall impedance for metallic multilayer tubes. Thus, the longitudinal impedance of arbitrary multilayers can always be split into two parts: the space charge impedance and the resistive-wall impedance. Theoretically, we can extend the result to any material of layers, as long as the first layer is metallic, which relates to $\kappa$.

The transverse impedance of the multilayer beam pipe can be obtained in the similar way. The corresponding longitudinal impedance of the dipole mode is (see Appendix B)

$$
Z_{\|}^{(1)}(\omega)=\frac{i c^{2} k_{r}^{3} \mu_{0}}{2 \pi r \omega}\left(1+\frac{k_{r}^{2} a_{1}^{2}}{8}\right)\left(\frac{p_{s}}{q_{s}} I_{1}\left(k_{r} r\right)+K_{1}\left(k_{r} r\right)\right),
$$

where the ratio of $p_{s} / q_{s}$ can be obtained from the expression

$$
\begin{aligned}
\left(\begin{array}{c}
p_{+} \\
p_{s}
\end{array}\right)_{1}= & {\left[\xi_{1} B^{*}\left(k_{r} a_{2}\right) N_{1}+\zeta_{1} D^{*}\left(k_{r} a_{2}\right) N_{2}\right] } \\
& \times\left[\xi_{1} A^{*}\left(k_{r} a_{2}\right) N_{1}+\zeta_{1} C^{*}\left(k_{r} a_{2}\right) N_{2}\right]^{-1}\left(\begin{array}{c}
0 \\
q_{s}
\end{array}\right)_{1}
\end{aligned}
$$

with

$$
\begin{aligned}
& N_{1}=\left(\begin{array}{ll}
L_{11}{ }^{(1)} & L_{12}{ }^{(1)}
\end{array}\right) \prod_{i=2}^{n-1}\left(\begin{array}{ll}
L_{11}{ }^{(i)} & L_{12}{ }^{(i)} \\
L_{21}{ }^{(i)} & L_{22}{ }^{(i)}
\end{array}\right)\left(\begin{array}{c}
B\left(\lambda_{n} a_{n}\right) \\
D\left(\lambda_{n} a_{n}\right)
\end{array}\right), \\
& N_{2}=\left(\begin{array}{ll}
L_{21}{ }^{(1)} & L_{22}{ }^{(1)}
\end{array}\right) \prod_{i=2}^{n-1}\left(\begin{array}{ll}
L_{11}{ }^{(i)} & L_{12}{ }^{(i)} \\
L_{21}{ }^{(i)} & L_{22}{ }^{(i)}
\end{array}\right)\left(\begin{array}{l}
B\left(\lambda_{n} a_{n}\right) \\
D\left(\lambda_{n} a_{n}\right)
\end{array}\right) .
\end{aligned}
$$

Comparing with the results in Ref. [8] obtained by Zotter and Kheifets, who also studied the impedance of multilayer pipes, we find that Eqs. (100)-(101) in Ref. [8] can be written as 


$$
\begin{aligned}
Z_{\|}(\omega)= & \frac{i Z_{0} c}{\pi \omega r^{2}}\left[\left[1-2 I_{1}\left(k_{r} r\right) K_{1}\left(k_{r} r\right)\right]+2 I_{1}^{2}\left(k_{r} r\right)\right. \\
& \left.\times \frac{Y_{1} K_{1}\left(k_{r} a_{2}\right)-K_{0}\left(k_{r} a_{2}\right)}{Y_{1} I_{1}\left(k_{r} a_{2}\right)+I_{0}\left(k_{r} a_{2}\right)}\right]
\end{aligned}
$$

if the transverse size $a$ of a uniform beam equals $r$. Like that in Ref. [8], $Y_{1}$ in Eq. (70) is determined as

$$
\begin{gathered}
Y_{i-1}=-X_{i} \frac{R_{i}+Y_{i} U_{i}}{R_{i}^{\prime}+Y_{i} U_{i}^{\prime}}, \quad Y_{n-1}=X_{n} \frac{K_{0}\left(\lambda_{n} a_{n-1}\right)}{K_{1}\left(\lambda_{n} a_{n-1}\right)}, \\
X_{i}=\frac{\lambda_{i} \varepsilon_{i-1}^{\prime}}{\varepsilon_{i}^{\prime} \lambda_{i-1}}
\end{gathered}
$$

with

$$
\begin{aligned}
& R_{i}\left(\lambda_{i} r\right)=K_{0}\left(\lambda_{i} a_{i}\right) I_{0}\left(\lambda_{i} r\right)-I_{0}\left(\lambda_{i} a_{i}\right) K_{0}\left(\lambda_{i} r\right), \\
& U_{i}\left(\lambda_{i} r\right)=-K_{1}\left(\lambda_{i} a_{i}\right) I_{0}\left(\lambda_{i} r\right)-I_{1}\left(\lambda_{i} a_{i}\right) K_{0}\left(\lambda_{i} r\right),
\end{aligned}
$$

and $R_{i}^{\prime}, U_{i}^{\prime}$ are the derivatives with respect to the argument. If $Y_{1}$ is replaced with $\kappa M$, which is used here, we can get the following expression of the longitudinal impedance of the multilayer pipes when we expand Eq. (70) up to first order in $\delta_{\text {skin }} \omega / c$ and second order in $k_{r} c / \omega$ :

TABLE I. Main parameters of three proton rings.

\begin{tabular}{lccc}
\hline \hline & CSNS & J-PARC & SNS \\
\hline$\gamma$ & 1.1 & 1.4 & 2.1 \\
$a_{2}(\mathrm{~cm})$ & 11 & 12.5 & 8 \\
$d_{1}=a_{3}-a_{2}(\mathrm{~nm})$ & 10 & 15 & 100 \\
$d_{2}=a_{4}-a_{3}(\mathrm{~mm})$ & 5 & 5 & 5 \\
\hline \hline
\end{tabular}

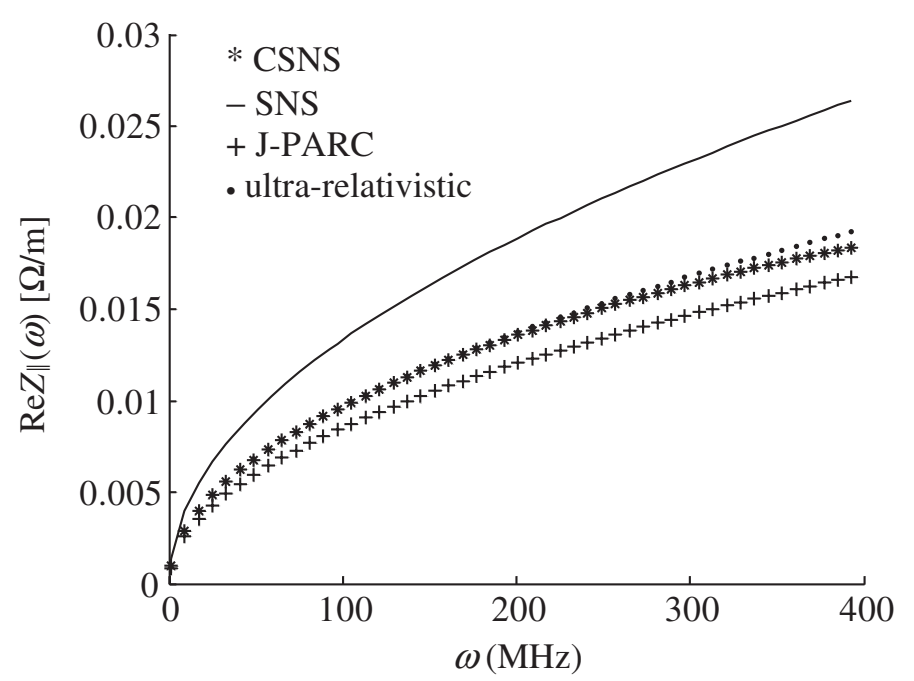

$$
\begin{aligned}
Z_{\|}(\omega)= & \frac{Z_{0}}{2 \pi c}\left[-\frac{i c^{2} k_{r}^{2}}{\omega} \ln \left(\frac{r}{a_{2}}\right)+\frac{\delta_{2} \mu_{2} \omega[\operatorname{sgn}(\omega)-i]}{2 a_{2} \mu_{0}}\right. \\
& \left.\times\left(1-\frac{k_{r}^{2}\left(2 a_{2}^{2}-r^{2}\right)}{4}\right) M\right] .
\end{aligned}
$$

Equation (73) looks much like our expression of Eq. (65), but missing a term $-a_{1}^{2}$ on the numerator in the parentheses of the second term. This is because we use a ring beam here, but a uniform round beam with a radius $a$ is taken in Ref. [8].

\section{APPLICATIONS TO REAL MACHINES}

Several examples of typical machines will be given in this section. Three proton rings, the rapid cycling synchrotrons of the China Spallation Neutron Source (CSNS) [12] and the Japan Proton Accelerator Research Complex (JPARC) [13], and the accumulator of the Spallation Neutron Source (SNS) [14] in the U.S.A., are evaluated here. The related parameters used in numerical estimations are listed in Table I.

We consider a stainless steel beam pipe with TiN coating inside, and the conductivity of TiN is $\sigma_{\text {TiN }}=5.88 \times$ $10^{6} \Omega^{-1} \mathrm{~m}^{-1}$. In all cases, we suppose the thickness of stainless steel beam pipe is $5 \mathrm{~mm}$ with a conductivity of $\sigma_{\mathrm{SS}}=1.4 \times 10^{6} \Omega^{-1} \mathrm{~m}^{-1}$. Figure 2 presents the real part of the longitudinal and transverse impedance of each case at a radius $r=a_{1}=a_{2} / 2$. The horizontal axis is the angular frequency with the range from 0 to $400 \mathrm{MHz}$.

From the numerical estimation based on the impedance expressions we got here, we can see that the longitudinal impedance varies both with the beam energy and the inner radius of the beam pipe. We also compare the result of the CSNS ring with the relativistic limit, and it is easy to tell the differences between them, especially the real part of transverse impedance.

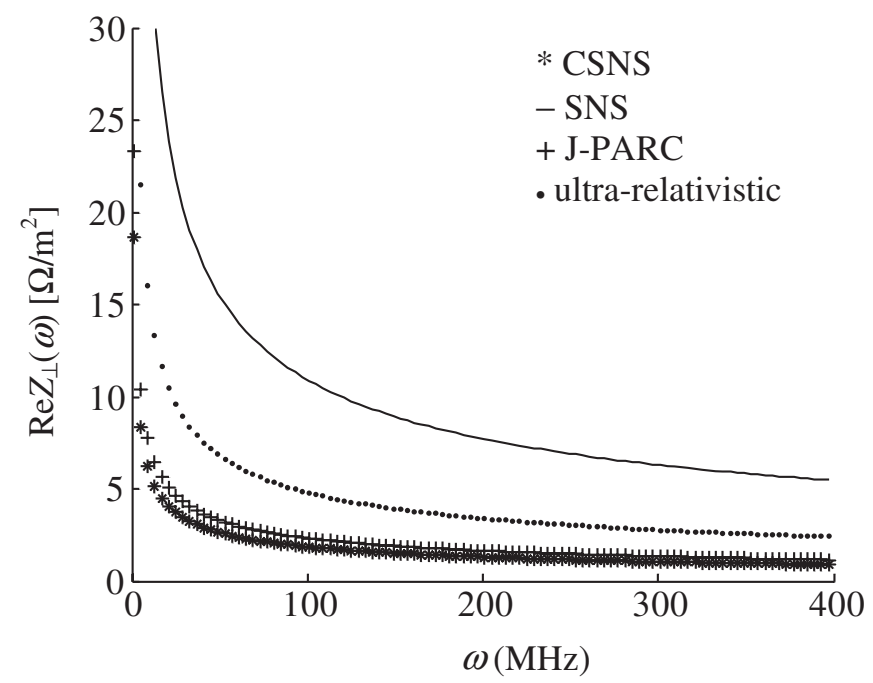

FIG. 2. Real part of longitudinal (left) and transverse (right) impedance per unit length for typical machines. 

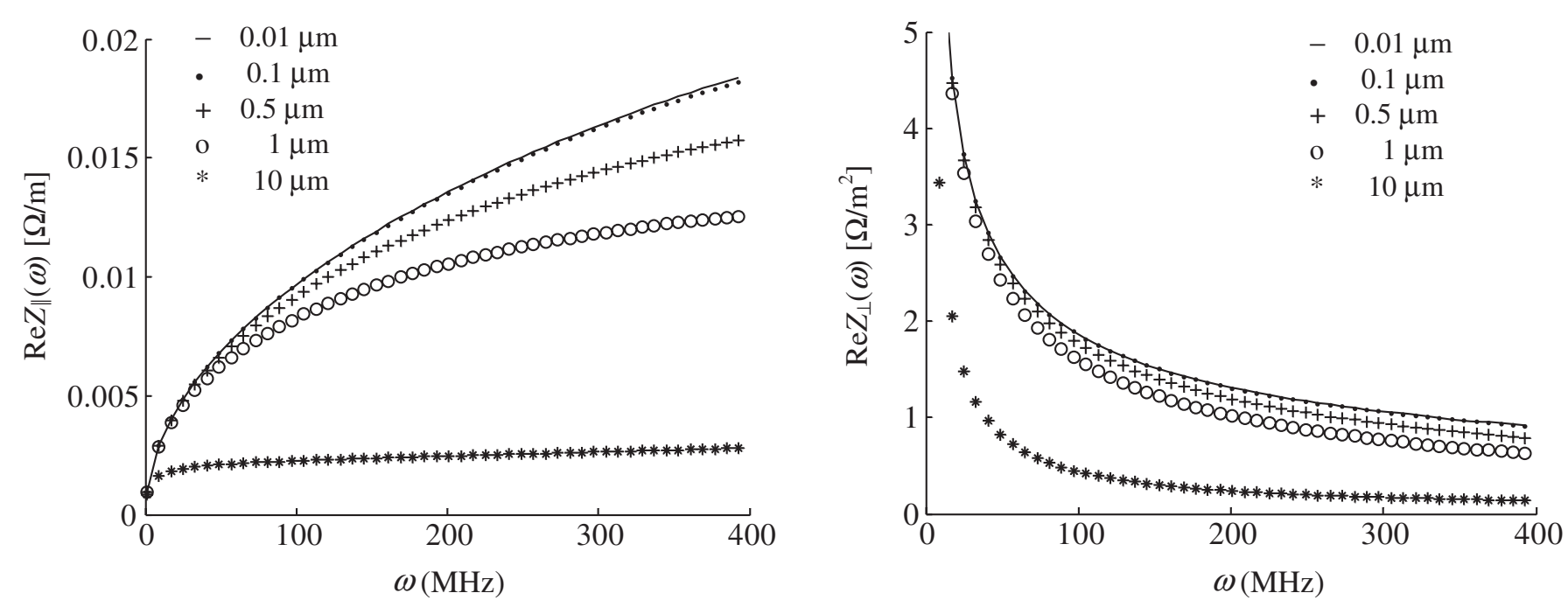

FIG. 3. Real part of longitudinal (left) and transverse (right) impedance per unit length for different thickness of inner layer (CSNS ring).

Figure 3 presents the real part of the resistive-wall impedance per unit length for different thicknesses of the cover layer using the CSNS parameters. Here a stainless steel beam pipe with a copper layer inside is considered. The conductivity of copper is $\sigma_{\mathrm{Cu}}=5.88 \times 10^{7} \Omega^{-1} \mathrm{~m}^{-1}$. The left figure denotes the longitudinal impedance, and the right one the transverse impedance.

The figure shows that, in the two-layer case, the clear difference appears at a thickness bigger than $\sim 100 \mathrm{~nm}$, and that the thicker the cover layer, the less the impedance. It is easy to understand that the thicker cover layer will contribute more to the total impedance as long as the cover thickness is smaller than the skin depth. Once the cover thickness is larger than the skin depth, the total impedance mainly comes from the cover layer.

\section{SUMMARY}

The general expressions of longitudinal and transverse impedance for two-layer tubes with finite thickness for a ring beam in arbitrary velocity have been obtained. The results can be extended to the case of multilayers.

As a starting point of calculation, the wave equation of scalar and vector potential is used, and the field matching method is applied. The results can be applicable to any material which obeys Ohm's law with a constant scalar conductivity, and that the dielectric constant and the magnetic permeability can be simply described as $\varepsilon=\varepsilon_{r} \varepsilon_{0}$, $\mu=\mu_{r} \mu_{0}$. As the impedance expressions in this report are derived for a ring beam of finite radius $a_{1}$, the results are not valid to the case of beam with $\rho \neq 0$ for $0 \leq r \leq$ $a_{1}$.

Meanwhile, we have given a compact expression of longitudinal and transverse impedance for the special case of the metallic two-layer tube. They can always be composed to two parts: the space charge impedance, which is independent on skin depth $\delta_{\text {skin }}$, and the resistive-wall impedance, which is proportional to $\delta_{\text {skin }}$. Our main results include the longitudinal impedance for the resistive wall given in Eq. (54):

$$
\begin{aligned}
Z_{\|}(\omega)= & \frac{Z_{0}}{2 \pi c}\left[-\frac{i c^{2} k_{r}^{2}}{\omega} \ln \left(\frac{r}{a_{2}}\right)+\frac{\delta_{2} \mu_{2} \omega[\operatorname{sgn}(\omega)-i]}{2 a_{2} \mu_{0}}\right. \\
& \left.\times\left(1-\frac{k_{r}^{2}\left(2 a_{2}^{2}-r^{2}-a_{1}^{2}\right)}{4}\right) M\right]
\end{aligned}
$$

with

$$
M=-\frac{\varepsilon_{0} \lambda_{3}\left[\alpha \tanh \left(x_{1}\right)+\tanh \left(x_{2}\right)\right] K_{1}\left(k_{r} a_{4}\right)+\varepsilon_{3}^{\prime} k_{r}\left[\alpha \tanh \left(x_{1}\right) \tanh \left(x_{2}\right)+1\right] K_{0}\left(k_{r} a_{4}\right)}{\varepsilon_{0} \lambda_{3}\left[\alpha+\tanh \left(x_{1}\right) \tanh \left(x_{2}\right)\right] K_{1}\left(k_{r} a_{4}\right)+\varepsilon_{3}^{\prime} k_{r}\left[\alpha \tanh \left(x_{2}\right)+\tanh \left(x_{1}\right)\right] K_{0}\left(k_{r} a_{4}\right)}
$$

and

$$
\kappa=-\frac{\lambda_{2}}{k_{r} \mu_{0} \varepsilon_{2}^{\prime} c^{2}},
$$

and the transverse impedance for the resistive wall given in Eq. (59), 


$$
Z_{\perp, r w}(\omega) \approx \frac{1}{\sqrt{\omega^{2} / c^{2}+k_{r}^{2}}} \frac{1-i \operatorname{sgn}(\omega)}{4 \pi a_{2}^{3} \delta_{2} \sigma_{2}}\left(4-k_{r}^{2} a_{2}^{2}+\frac{1}{2} k_{r}^{2} r^{2}+\frac{1}{2} k_{r}^{2} a_{1}^{2}\right) \frac{1+\alpha \tanh \left(x_{1}\right) \tanh \left(x_{2}\right)}{\alpha \tanh \left(x_{2}\right)+\tanh \left(x_{1}\right)}
$$

For both cases, we can generalize the results to the impedance of the one-layer tube, which are consistent with that derived in Ref. [1]. In the ultrarelativistic limit, the longitudinal impedance we got can be reduced to the results obtained by Ivanyan [3]. All the results can be deduced to the point beam case.

For the case of a metallic multilayer, the longitudinal impedance has the same expression as the two-layer tube Eq. (54), except for $M$, which is given in Eq. (66). The results agree well enough with those obtained by Zotter and Kheifets in Ref. [8] if we expand our results to the first order in $\delta_{\text {skin }} \omega / c$ and to the second order of $k_{r} c / \omega$.

The numerical estimations on different hadron machines also show that the nonrelativistic formulas of the impedance deviate from those of the ultrarelativistic limit. For the longitudinal impedance, the real part for different beam energy departs far as the beam frequency increases, though at the low frequency they seem to merge. When some instabilities happen, the growth time will decrease since the integration of the real part of impedance at lower energy will be smaller than that of the cases for ultrarelativistic or relatively higher beam energy. In the two-layer case, the impedance deviates more when the thickness of the inner coating increases.

\section{ACKNOWLEDGMENTS}

The authors would like to thank the AP group of CSNS for discussion and also D.M. Zhou for providing Refs. [5,6]. Special thanks are given to F. Zimmermann and K. Oide for their discussions on the results in Ref. [1]. This work was supported by the Knowledge Innovation Program of the Chinese Academy of Sciences, and partly by the JSPS Core University Program.

\section{APPENDIX A}

By using the field matching method, we can obtain the unknown coefficients in the wakefield expression, Eq. (10). The field in the interface of two different layers satisfies the condition of Eq. (15). For an $n$-layers tube (here $n$ including the vacuum layer and $n>1$ ), the coefficient $p_{1}$ can be obtained from the matching condition,

$$
\left(\begin{array}{c}
p \\
q
\end{array}\right)_{1}=\prod_{i=1}^{n-1} F^{-1}\left(\lambda_{i} a_{i+1}\right) F\left(\lambda_{i+1} a_{i+1}\right)\left(\begin{array}{c}
p \\
q
\end{array}\right)_{n}
$$

in which $q_{1}$ is defined by Eq. (21) and $p_{n}=0$. The condition (A1) can also be expressed as

$$
\begin{aligned}
\left(\begin{array}{l}
p \\
q
\end{array}\right)_{1}= & F^{-1}\left(\lambda_{1} a_{2}\right) \\
& \times\left(\prod_{i=2}^{n-1} F\left(\lambda_{i} a_{i}\right) F^{-1}\left(\lambda_{i} a_{i+1}\right)\right) F\left(\lambda_{n} a_{n}\right)\left(\begin{array}{l}
p \\
q
\end{array}\right)_{n},
\end{aligned}
$$

with

$$
F\left(\lambda_{i} a_{i}\right) F^{-1}\left(\lambda_{i} a_{i+1}\right)=\left(\begin{array}{cc}
-\frac{b_{i}}{b_{i+1}} S_{i, 1} & \frac{b_{i}}{c_{i+1}} S_{i, 2} \\
\frac{c_{i}}{b_{i+1}} S_{i, 3} & -\frac{c_{i}}{c_{i+1}} S_{i, 4}
\end{array}\right)
$$

and

$S_{i, 1}=c_{i} b_{i+1}\left[I_{1}\left(\lambda_{i} a_{i}\right) K_{0}\left(\lambda_{i} a_{i+1}\right)+K_{1}\left(\lambda_{i} a_{i}\right) I_{0}\left(\lambda_{i} a_{i+1}\right)\right]$,

$S_{i, 2}=b_{i} c_{i+1}\left[I_{1}\left(\lambda_{i} a_{i}\right) K_{1}\left(\lambda_{i} a_{i+1}\right)-K_{1}\left(\lambda_{i} a_{i}\right) I_{1}\left(\lambda_{i} a_{i+1}\right)\right]$,

$S_{i, 3}=c_{i} b_{i+1}\left[I_{0}\left(\lambda_{i} a_{i}\right) K_{0}\left(\lambda_{i} a_{i+1}\right)-K_{0}\left(\lambda_{i} a_{i}\right) I_{0}\left(\lambda_{i} a_{i+1}\right)\right]$,

$S_{i, 4}=b_{i} c_{i+1}\left[I_{0}\left(\lambda_{i} a_{i}\right) K_{1}\left(\lambda_{i} a_{i+1}\right)+K_{0}\left(\lambda_{i} a_{i}\right) I_{1}\left(\lambda_{i} a_{i+1}\right)\right]$.

If we further represent the product of matrices as

$$
\begin{aligned}
\left(\begin{array}{ll}
l_{11} & l_{12} \\
l_{21} & l_{22}
\end{array}\right) & =\prod_{i=2}^{n-1} F\left(\lambda_{i} a_{i}\right) F^{-1}\left(\lambda_{i} a_{i+1}\right) \\
& =\prod_{i=2}^{n-1}\left(\begin{array}{cc}
-\frac{b_{i}}{b_{i+1}} S_{i, 1} & \frac{b_{i}}{c_{i+1}} S_{i, 2} \\
\frac{c_{i}}{b_{i+1}} S_{i, 3} & -\frac{c_{i}}{c_{i+1}} S_{i, 4}
\end{array}\right),
\end{aligned}
$$

then we have

$$
\begin{aligned}
\left(\begin{array}{c}
p \\
q
\end{array}\right)_{1}= & -\frac{\lambda_{1} a_{2}}{b_{1} c_{1}}\left(\begin{array}{cc}
c_{1} K_{0}\left(\lambda_{1} a_{2}\right) & -b_{1} K_{1}\left(\lambda_{1} a_{2}\right) \\
-c_{1} I_{0}\left(\lambda_{1} a_{2}\right) & -b_{1} I_{1}\left(\lambda_{1} a_{2}\right)
\end{array}\right) \\
& \times\left(\begin{array}{cc}
l_{11} & l_{12} \\
l_{21} & l_{22}
\end{array}\right) \\
& \times\left(\begin{array}{cc}
-b_{n} I_{1}\left(\lambda_{n} a_{n}\right) & b_{n} K_{1}\left(\lambda_{n} a_{n}\right) \\
c_{n} I_{0}\left(\lambda_{n} a_{n}\right) & c_{n} K_{0}\left(\lambda_{n} a_{n}\right)
\end{array}\right)\left(\begin{array}{c}
p \\
q
\end{array}\right)_{n} .
\end{aligned}
$$

Considering that $p_{n}=0$ in the vacuum outside the beam chamber, then the ratio of $p_{1}$ and $q_{1}$ will be

$$
\frac{p_{1}}{q_{1}}=\frac{\kappa M K_{1}\left(\lambda_{1} a_{2}\right)-K_{0}\left(\lambda_{1} a_{2}\right)}{\kappa M I_{1}\left(\lambda_{1} a_{2}\right)+I_{0}\left(\lambda_{1} a_{2}\right)},
$$

where

$$
\lambda_{1}=k_{r}, \quad \kappa=-\frac{b_{1} c_{2}}{c_{1} b_{2}},
$$


and

$$
\begin{aligned}
& M=-\frac{b_{2}}{c_{2}} \frac{c_{n} l_{22} K_{0}\left(\lambda_{n} a_{n}\right)+b_{n} l_{21} K_{1}\left(\lambda_{n} a_{n}\right)}{c_{n} l_{12} K_{0}\left(\lambda_{n} a_{n}\right)+b_{n} l_{11} K_{1}\left(\lambda_{n} a_{n}\right)}
\end{aligned}
$$

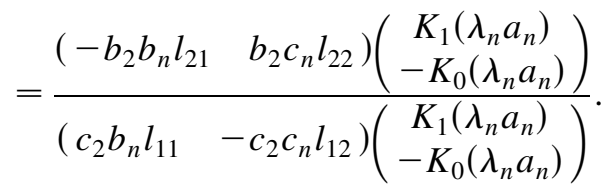

For $n=3$, i.e., the case of a one-layer tube of finite thickness,

$$
\left(\begin{array}{ll}
l_{11} & l_{12} \\
l_{21} & l_{22}
\end{array}\right)=\left(\begin{array}{cc}
-\frac{b_{2}}{b_{3}} S_{2,1} & \frac{b_{2}}{c_{3}} S_{2,2} \\
\frac{c_{2}}{b_{3}} S_{2,3} & -\frac{c_{2}}{c_{3}} S_{2,4}
\end{array}\right),
$$

and

$$
M=\frac{\left(\begin{array}{ll}
S_{2,3} & S_{2,4}
\end{array}\right)\left(\begin{array}{c}
K_{1}\left(\lambda_{3} a_{3}\right) \\
-K_{0}\left(\lambda_{3} a_{3}\right)
\end{array}\right)}{\left(\begin{array}{ll}
S_{2,1} & S_{2,2}
\end{array}\right)\left(\begin{array}{c}
K_{1}\left(\lambda_{3} a_{3}\right) \\
-K_{0}\left(\lambda_{3} a_{3}\right)
\end{array}\right)}
$$

For $n>3$,

$$
\begin{aligned}
& b_{2} b_{n} l_{21}=b_{2} c_{2}\left(\frac{1}{b_{3}} S_{2,3} \quad-\frac{1}{c_{3}} S_{2,4}\right) \\
& \times \prod_{i=3}^{n-2}\left(\begin{array}{cc}
-b_{i} S_{i, 1} / b_{i+1} & b_{i} S_{i, 2} / c_{i+1} \\
c_{i} S_{i, 3} / b_{j+1} & -c_{i} S_{i, 4} / c_{i+1}
\end{array}\right) \\
& \times\left(\begin{array}{c}
-b_{n-1} S_{n-1,1} \\
c_{n-1} S_{n-1,3}
\end{array}\right), \\
& b_{2} c_{n} l_{22}=-b_{2} c_{2}\left(\frac{1}{b_{3}} S_{2,3}-\frac{1}{c_{3}} S_{2,4}\right) \\
& \times \prod_{i=3}^{n-2}\left(\begin{array}{cc}
-b_{i} S_{i, 1} / b_{i+1} & b_{i} S_{i, 2} / c_{i+1} \\
c_{i} S_{i, 3} / b_{i+1} & -c_{i} S_{i, 4} / c_{i+1}
\end{array}\right) \\
& \times\left(\begin{array}{c}
-b_{n-1} S_{n-1,2} \\
c_{n-1} S_{n-1,4}
\end{array}\right), \\
& c_{2} b_{n} l_{11}=-b_{2} c_{2}\left(-\frac{1}{b_{3}} S_{2,1} \quad \frac{1}{c_{3}} S_{2,2}\right) \\
& \times \prod_{i=3}^{n-2}\left(\begin{array}{cc}
-b_{i} S_{i, 1} / b_{i+1} & b_{i} S_{i, 2} / c_{i+1} \\
c_{i} S_{i, 3} / b_{i+1} & -c_{i} S_{i, 4} / c_{i+1}
\end{array}\right) \\
& \times\left(\begin{array}{c}
b_{n-1} S_{n-1,1} \\
-c_{n-1} S_{n-1,3}
\end{array}\right), \\
& c_{2} c_{n} l_{12}=b_{2} c_{2}\left(-\frac{1}{b_{3}} S_{2,1} \quad \frac{1}{c_{3}} S_{2,2}\right) \\
& \times \prod_{i=3}^{n-2}\left(\begin{array}{cc}
-b_{i} S_{i, 1} / b_{i+1} & b_{i} S_{i, 2} / c_{i+1} \\
c_{i} S_{i, 3} / b_{i+1} & -c_{i} S_{i, 4} / c_{i+1}
\end{array}\right) \\
& \times\left(\begin{array}{c}
b_{n-1} S_{n-1,2} \\
-c_{n-1} S_{n-1,4}
\end{array}\right) \text {. }
\end{aligned}
$$

By expanding the expressions above, we find that the coefficients in front of $S$ 's in the matrices will cancel each other, and the expressions are simplified to

$$
\begin{aligned}
& \left(\begin{array}{ll}
-b_{2} b_{n} l_{21} & b_{2} c_{n} l_{22}
\end{array}\right)=-b_{2} c_{2}\left(\begin{array}{ll}
S_{2,3} & S_{2,4}
\end{array}\right) \\
& \times \prod_{i=3}^{n-1}\left(\begin{array}{ll}
S_{i, 1} & S_{i, 2} \\
S_{i, 3} & S_{i, 4}
\end{array}\right),
\end{aligned}
$$

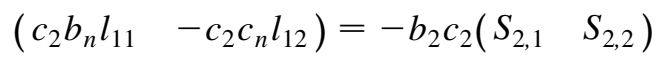

$$
\begin{aligned}
& \times \prod_{i=3}^{n-1}\left(\begin{array}{cc}
S_{i, 1} & S_{i, 2} \\
S_{i, 3} & S_{i, 4}
\end{array}\right) .
\end{aligned}
$$

Now we take the above expressions into (A9), then $M$ will become

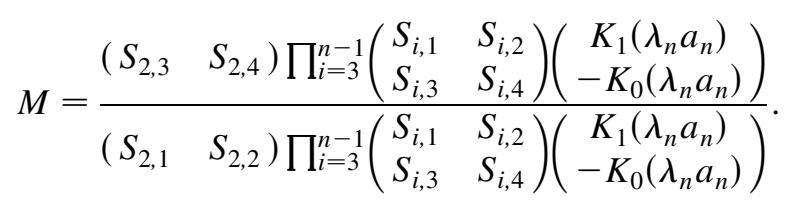

For completeness, we take

$$
\left(\begin{array}{ll}
S_{1,1} & S_{1,2} \\
S_{1,3} & S_{1,4}
\end{array}\right)=\left(\begin{array}{ll}
1 & 0 \\
0 & 1
\end{array}\right)
$$

which corresponds to the case of $n=2$. Then $M$ has a generalized form of

$$
M=\frac{\left(\begin{array}{ll}
S_{1,3} & S_{1,4}
\end{array}\right) \prod_{i=2}^{n-1}\left(\begin{array}{ll}
S_{i, 1} & S_{i, 2} \\
S_{i, 3} & S_{i, 4}
\end{array}\right)\left(\begin{array}{c}
K_{1}\left(\lambda_{n} a_{n}\right) \\
-K_{0}\left(\lambda_{n} a_{n}\right)
\end{array}\right)}{\left(\begin{array}{ll}
S_{1,1} & S_{1,2}
\end{array}\right) \prod_{i=2}^{n-1}\left(\begin{array}{ll}
S_{i, 1} & S_{i, 2} \\
S_{i, 3} & S_{i, 4}
\end{array}\right)\left(\begin{array}{c}
K_{1}\left(\lambda_{n} a_{n}\right) \\
-K_{0}\left(\lambda_{n} a_{n}\right)
\end{array}\right)} .
$$

Now that we have obtained the expression of $\kappa$ and $M$, the ratio of $p_{1}$ and $q_{1}$ can be obtained by Eq. (A7). Correspondingly, the wakefield and impedance of the monopolar mode can be derived, in the respect that the parameter $q_{1}$ is determined by the source boundary.

\section{APPENDIX B}

In deriving the transverse impedance, the unknown coefficients are determined by

$$
\left(\begin{array}{c}
p_{+} \\
p_{s} \\
0 \\
q_{s}
\end{array}\right)_{1}=\prod_{i=1}^{n-1} G^{-1}\left(\lambda_{i} a_{i+1}\right) G\left(\lambda_{i+1} a_{i+1}\right)\left(\begin{array}{c}
0 \\
0 \\
q_{+} \\
q_{s}
\end{array}\right)_{n} .
$$

By decomposing the matrices $G$ and $G^{-1}$ into $2 \times 2$ block matrices,

$$
\begin{aligned}
G\left(\lambda_{i} r\right) & =\left(\begin{array}{ll}
A\left(\lambda_{i} r\right) & B\left(\lambda_{i} r\right) \\
C\left(\lambda_{i} r\right) & D\left(\lambda_{i} r\right)
\end{array}\right), \\
G^{-1}\left(\lambda_{i} r\right) & =\left(\begin{array}{ll}
\xi_{i} B^{*}\left(\lambda_{i} r\right) & \zeta_{i} D^{*}\left(\lambda_{i} r\right) \\
\xi_{i} A^{*}\left(\lambda_{i} r\right) & \zeta_{i} C^{*}\left(\lambda_{i} r\right)
\end{array}\right),
\end{aligned}
$$


and further defining

$$
\left(\begin{array}{ll}
L_{11}{ }^{(i)} & L_{12}{ }^{(i)} \\
L_{21}{ }^{(i)} & L_{22}{ }^{(i)}
\end{array}\right)=G\left(\lambda_{i} a_{i}\right) G^{-1}\left(\lambda_{i} a_{i+1}\right),
$$

with

$$
\begin{aligned}
L_{11}{ }^{(i)}= & \left(\begin{array}{cc}
\lambda_{i} a_{i+1} T_{i, 1} & \frac{i k\left(a_{i} T_{i, 1}+a_{i+1} T_{i, 2}\right)}{\lambda_{i} a_{i}} \\
0 & -\lambda_{i} a_{i+1} T_{i, 2}
\end{array}\right), \\
L_{12}{ }^{(i)}= & \frac{1}{\mu_{i} \omega} \\
& \times\left(\begin{array}{cc}
-\frac{a_{i+1}}{a_{i}} k T_{i, 3} & i\left(\frac{k^{2} T_{i, 3}}{a_{i} \lambda_{i}^{2}}+a_{i+1}\left(k^{2}-\lambda_{i}^{2}\right) T_{i, 4}\right) \\
-i a_{i+1} \lambda_{i}^{2} T_{i, 3} & -k T_{i, 3}
\end{array}\right), \\
L_{21}{ }^{(i)}= & \frac{\mu_{i} \omega}{\left(k^{2}-\lambda_{i}^{2}\right)} \\
& \times\left(\begin{array}{cc}
-\frac{a_{i+1}}{a_{i}} k T_{i, 3} & -i\left(\frac{k^{2} T_{i, 3}}{a_{i} \lambda_{i}^{2}}+a_{i+1}\left(k^{2}-\lambda_{i}^{2}\right) T_{i, 4}\right) \\
i a_{i+1} \lambda_{i}^{2} T_{i, 3} & -k T_{i, 3}
\end{array}\right), \\
L_{22}{ }^{(i)}= & \left(\begin{array}{cc}
\lambda_{i} a_{i+1} T_{i, 1} & -\frac{i k\left(a_{i} T_{i, 1}+a_{i+1} T_{i, 2}\right)}{\lambda_{i} a_{i}} \\
0 & -\lambda_{i} a_{i+1} T_{i, 2}
\end{array}\right),
\end{aligned}
$$

where

$$
\begin{aligned}
& T_{i, 1}=I_{1}^{\prime}\left(\lambda_{i} a_{i}\right) K_{1}\left(\lambda_{i} a_{i+1}\right)-I_{1}\left(\lambda_{i} a_{i+1}\right) K_{1}^{\prime}\left(\lambda_{i} a_{i}\right), \\
& T_{i, 2}=I_{1}\left(\lambda_{i} a_{i}\right) K_{1}^{\prime}\left(\lambda_{i} a_{i+1}\right)-I_{1}^{\prime}\left(\lambda_{i} a_{i+1}\right) K_{1}\left(\lambda_{i} a_{i}\right), \\
& T_{i, 3}=I_{1}\left(\lambda_{i} a_{i}\right) K_{1}\left(\lambda_{i} a_{i+1}\right)-I_{1}\left(\lambda_{i} a_{i+1}\right) K_{1}\left(\lambda_{i} a_{i}\right), \\
& T_{i, 4}=I_{1}^{\prime}\left(\lambda_{i} a_{i}\right) K_{1}^{\prime}\left(\lambda_{i} a_{i+1}\right)-I_{1}^{\prime}\left(\lambda_{i} a_{i+1}\right) K_{1}^{\prime}\left(\lambda_{i} a_{i}\right),
\end{aligned}
$$

we have

$$
\begin{aligned}
\left(\begin{array}{c}
p_{+} \\
p_{s} \\
0 \\
q_{s}
\end{array}\right)_{1}= & \left(\begin{array}{ll}
\xi_{1} B^{*}\left(k_{r} a_{2}\right) & \zeta_{1} D^{*}\left(k_{r} a_{2}\right) \\
\xi_{1} A^{*}\left(k_{r} a_{2}\right) & \zeta_{1} C^{*}\left(k_{r} a_{2}\right)
\end{array}\right) \\
& \times \prod_{i=2}^{n-1}\left(\begin{array}{ll}
L_{11}{ }^{(i)} & L_{12}{ }^{(i)} \\
L_{21}{ }^{(i)} & L_{22}{ }^{(i)}
\end{array}\right) \\
& \times\left(\begin{array}{ll}
A\left(\lambda_{n} a_{n}\right) & B\left(\lambda_{n} a_{n}\right) \\
C\left(\lambda_{n} a_{n}\right) & D\left(\lambda_{n} a_{n}\right)
\end{array}\right)\left(\begin{array}{c}
0 \\
0 \\
q_{+} \\
q_{s}
\end{array}\right)_{n}
\end{aligned}
$$

Since Eq. (B6) can be transformed to

$$
\begin{aligned}
\left(\begin{array}{c}
p_{+} \\
p_{s}
\end{array}\right)_{1}= & {\left[\begin{array}{ll}
\xi_{1} B^{*}\left(k_{r} a_{2}\right) & \zeta_{1} D^{*}\left(k_{r} a_{2}\right)
\end{array}\right] } \\
& \times \prod_{i=2}^{n-1}\left(\begin{array}{ll}
L_{11}{ }^{(i)} & L_{12}{ }^{(i)} \\
L_{21}{ }^{(i)} & L_{22}{ }^{(i)}
\end{array}\right)\left(\begin{array}{c}
B\left(\lambda_{n} a_{n}\right) \\
D\left(\lambda_{n} a_{n}\right)
\end{array}\right)\left(\begin{array}{c}
q_{+} \\
q_{s}
\end{array}\right)_{n},
\end{aligned}
$$

and

$$
\begin{aligned}
& \left(\begin{array}{c}
0 \\
q_{s}
\end{array}\right)_{1}=\left[\begin{array}{ll}
\xi_{1} A^{*}\left(k_{r} a_{2}\right) & \zeta_{1} C^{*}\left(k_{r} a_{2}\right)
\end{array}\right] \\
& \times \prod_{i=2}^{n-1}\left(\begin{array}{ll}
L_{11}{ }^{(i)} & L_{12}{ }^{(i)} \\
L_{21}{ }^{(i)} & L_{22}{ }^{(i)}
\end{array}\right)\left(\begin{array}{c}
B\left(\lambda_{n} a_{n}\right) \\
D\left(\lambda_{n} a_{n}\right)
\end{array}\right)\left(\begin{array}{c}
q_{+} \\
q_{s}
\end{array}\right)_{n},
\end{aligned}
$$

we have

$$
\begin{aligned}
\left(\begin{array}{c}
p_{+} \\
p_{s}
\end{array}\right)_{1}= & {\left[\xi_{1} B^{*}\left(k_{r} a_{2}\right) N_{1}+\zeta_{1} D^{*}\left(k_{r} a_{2}\right) N_{2}\right] } \\
& \times\left[\xi_{1} A^{*}\left(k_{r} a_{2}\right) N_{1}+\zeta_{1} C^{*}\left(k_{r} a_{2}\right) N_{2}\right]^{-1}\left(\begin{array}{c}
0 \\
q_{s}
\end{array}\right)_{1},
\end{aligned}
$$

where

$$
N_{1}=\left(L_{11}{ }^{(2)} \quad L_{12}{ }^{(2)}\right) \prod_{i=3}^{n-1}\left(\begin{array}{ll}
L_{11}{ }^{(i)} & L_{12}{ }^{(i)} \\
L_{21}{ }^{(i)} & L_{22}{ }^{(i)}
\end{array}\right)\left(\begin{array}{c}
B\left(\lambda_{n} a_{n}\right) \\
D\left(\lambda_{n} a_{n}\right)
\end{array}\right),
$$

and

$$
N_{2}=\left(\begin{array}{ll}
L_{21}{ }^{(2)} & L_{22}{ }^{(2)}
\end{array}\right) \prod_{i=3}^{n-1}\left(\begin{array}{ll}
L_{11}{ }^{(i)} & L_{12}{ }^{(i)} \\
L_{21}{ }^{(i)} & L_{22}{ }^{(i)}
\end{array}\right)\left(\begin{array}{c}
B\left(\lambda_{n} a_{n}\right) \\
D\left(\lambda_{n} a_{n}\right)
\end{array}\right) .
$$

For completeness, we further define

$$
\left(\begin{array}{ll}
L_{11}{ }^{(1)} & L_{12}{ }^{(1)} \\
L_{21}{ }^{(1)} & L_{22}{ }^{(1)}
\end{array}\right)=\left(\begin{array}{cc}
E & 0 \\
0 & E
\end{array}\right),
$$

where $E$ is the identity matrix. Correspondingly, the parameters $N_{1}$ and $N_{2}$ are reduced to

$$
\begin{array}{r}
N_{1}=\left(\begin{array}{ll}
L_{11}{ }^{(1)} & L_{12}{ }^{(1)}
\end{array}\right) \prod_{i=2}^{n-1}\left(\begin{array}{ll}
L_{11}{ }^{(i)} & L_{12}{ }^{(i)} \\
L_{21}{ }^{(i)} & L_{22}{ }^{(i)}
\end{array}\right)\left(\begin{array}{r}
B\left(\lambda_{n} a_{n}\right) \\
D\left(\lambda_{n} a_{n}\right)
\end{array}\right), \\
(\mathrm{B} 13 \\
N_{2}=\left(\begin{array}{ll}
L_{21}{ }^{(1)} & L_{22}{ }^{(1)}
\end{array}\right) \prod_{i=2}^{n-1}\left(\begin{array}{ll}
L_{11}{ }^{(i)} & L_{12}{ }^{(i)} \\
L_{21}{ }^{(i)} & L_{22}{ }^{(i)}
\end{array}\right)\left(\begin{array}{c}
B\left(\lambda_{n} a_{n}\right) \\
D\left(\lambda_{n} a_{n}\right)
\end{array}\right) .
\end{array}
$$


Then the ratio $p_{s} / q_{s}$ can be derived, and the longitudinal electric field of the dipolar mode is

$$
\begin{aligned}
E_{s} & =-\frac{i c^{2} k_{r}^{2}}{\omega} q_{s}\left(\frac{p_{s}}{q_{s}} I_{1}\left(k_{r} r\right)+K_{1}\left(k_{r} r\right)\right) \cos \varphi e^{i k z} \\
& =-\frac{i c^{2} k_{r}^{2}}{\omega} \frac{\mu_{0} \lambda_{b} v k_{r} d}{2 \pi}\left(\frac{p_{s}}{q_{s}} I_{1}\left(k_{r} r\right)+K_{1}\left(k_{r} r\right)\right) \cos \varphi e^{i k z}
\end{aligned}
$$

[1] F. Zimmermann and K. Oide, Phys. Rev. ST Accel. Beams 7, 044201 (2004).

[2] R. L. Gluckstern, CERN Report No. 2000-011, 2000.

[3] A. M. Al-khateeb, O. Boine-Frankenheim, R. W. Hasse, and I. Hofmann, Phys. Rev. E 71, 026501 (2005).

[4] A. M. Al-khateeb, R. W. Hasse, O. Boine-Frankenheim, W. M. Daqa, and I. Hofmann, Phys. Rev. ST Accel. Beams 10, 064401 (2007).

[5] M. Ivanyan and V. Tsakanov, Phys. Rev. ST Accel. Beams 7, 114402 (2004).
[6] M. Ivanyan and A. V. Tsakanian, Proceedings of the PAC 2005, Knoxville, Tennessee, 2005 (IEEE, Piscataway, New Jersey, 2005), p. 3138.

[7] M. Ivanyan and Andranik Tsakanian, in Phys. Rev. ST Accel. Beams 9, 034404 (2006).

[8] B. W. Zotter and S. A. Kheifets, Impedances and Wakes in High-Energy Particle Accelerators (World Scientific, Singapore, 1998).

[9] A. Chao, Physics of Collective Beam Instabilities in HighEnergy Particle Accelerators (Wiley, New York, 1993).

[10] L.C. Andrews, Special Functions for Engineers and Applied Mathematicians (Macmillan, New York, 1985).

[11] W. K. H. Panofsky and W. A. Wenzel, Rev. Sci. Instrum. 27, 967 (1956).

[12] CSNS Accelerator Team, IHEP-CSNS-Report/2004-01E, 2004.

[13] Y. Yamazaki et al., KEK, Report No. 2002-13, 2003; JAERI Report No. TECH-2003-044, 2003.

[14] J. Wei, in Proceedings of the Workshop on Space Charge Physics in High Intensity Hadron Rings, Shelter Island, New York, 1998, AIP Conf. Proc. No. 448 (AIP, New York, 1998), p. 152. 